\title{
Experimental approach to measure thick target neutron yields induced by heavy ions for shielding
}

\author{
N.D. Trinh ${ }^{1,{ }^{*}}$, M. Fadil ${ }^{1}{ }^{*}$, M. Lewitowicz ${ }^{1,{ }^{*}}$, C. Brouillard ${ }^{1}$, T. Clerc ${ }^{1}$, S. Damoy ${ }^{1}$, V. Desmezières ${ }^{1}$, E. Dessay ${ }^{1}$, M. Dupuis $^{1}$, \\ G.F. Grinyer ${ }^{1}$, J. Grinyer ${ }^{1}$, B.Jacquot ${ }^{1}$, X. Ledoux ${ }^{1}$, A. Madeline ${ }^{1}$, N. Menard ${ }^{1}$, M. Michel ${ }^{1}$, V. Morel ${ }^{1}$, F. Porée ${ }^{1}$, B. Rannou ${ }^{1}$ \\ and A. Savalle ${ }^{1}$ \\ ${ }^{1}$ Grand Accélérateur National d'Ions Lourds, Bd Henri Becquerel, 14076 Caen, France
}

\begin{abstract}
Double differential (angular and energy) neutron distributions were measured using an activation foil technique. Reactions were induced by impinging two low-energy heavy-ion beams accelerated with the GANIL CSS1 cyclotron: $\left({ }^{36} \mathrm{~S}(12 \mathrm{MeV} / \mathrm{u})\right.$ and $\left.{ }^{208} \mathrm{~Pb}(6.25 \mathrm{MeV} / \mathrm{u})\right)$ onto thick ${ }^{\text {nat }} \mathrm{Cu}$ targets. Results have been compared to Monte-Carlo calculations from two codes (PHITS and FLUKA) for the purpose of benchmarking radiation protection and shielding requirements. This comparison suggests a disagreement between calculations and experiment, particularly for high-energy neutrons.
\end{abstract}

\section{Introduction}

Nowadays, particle accelerators are used in many different fields from fundamental research to applications in medicine and in industry. In order to maintain a good level of knowledge concerning the radiation protection and shielding requirements and to answer to emerging needs in these fields with next-generation particle accelerators, characterization of potential radiation hazards like neutron yields induced by particle beams must be well known [1,2]. At GANIL, operation of its existing cyclotrons and the recent installation of a new high-intensity LINAC for SPIRAL 2 imply that any potential safety risk must be accurately evaluated.

Neutron yields are usually estimated from MonteCarlo transport codes such as FLUKA or PHITS. Due to the lack of the data concerning neutron yields induced by heavy-ion reactions around $10 \mathrm{MeV} / \mathrm{u}$ [1,2], we performed an experimental campaign at GANIL with the purpose of benchmarking the physics models in transport codes around this low energy limit.

\section{Experimental method}

\subsection{Experimental setup}

Two experiments were carried out in 2015 using the GANIL CSS1 cyclotron with two low-energy beams: ${ }^{36} \mathrm{~S}$ at $12 \mathrm{MeV} / \mathrm{u}$ and ${ }^{208} \mathrm{~Pb}$ at $6.25 \mathrm{MeV} / \mathrm{u}$.

Since copper is one of the prominent elements in accelerators and beam-line components, a thick ${ }^{\text {nat }} \mathrm{Cu}$ target was chosen to study neutron yields under nominal operating conditions. The target was $5 * 5 \mathrm{~cm}^{2}$ and had a thickness of $0.1 \mathrm{~cm}$ to completely stop the incident beams.

To measure neutrons, the activation-foil technique was employed. During the irradiation of the target, the resulting neutrons induced various threshold reactions in a series of activation foils that were placed around the target position (see Fig. 1). In this work, indium, nickel, cobalt, iron, aluminum, niobium and bismuth were chosen as activation foils. This choice permits neutron energies from $0.5 \mathrm{MeV}$ to $70 \mathrm{MeV}$ to be measured. The foils were $2.5 * 2.5 \mathrm{~cm}^{2}$ with a thickness of $0.1 \mathrm{~cm}$ in order to minimize the attenuation and the diffusion of neutrons. After irradiation of the ${ }^{\text {nat }} \mathrm{Cu}$ target, the measurement of the resulting activities in the activation foils was used to reconstruct the incident neutron spectra $[3,4,5]$ using an unfolding method.

The experimental setup is shown in Fig. 1. The ${ }^{\text {nat }} \mathrm{Cu}$ target is mounted on a $1.6 \mathrm{~cm}$ thick steel backing. On the other side of this backing, activation foils were placed from $0^{\circ}$ to $75^{\circ}$ on an aluminum support. At each position, different foils were stacked in a sandwich form.

\footnotetext{
To whom all correspondence should be addressed: trinh@ganil.fr, fadil@ganil.fr and lewitowicz@ganil.fr
} 


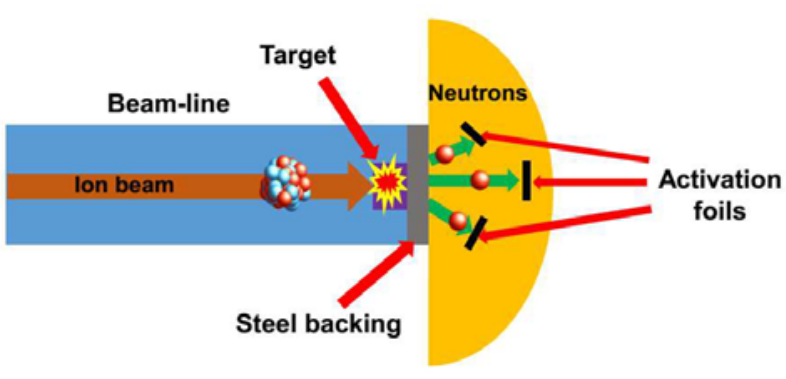

Figure 1: Experimental setup for the ion-beam irradiation and foil-activation measurements

In Table 1, a summary of the beam characteristics for both experiments is provided. During the irradiation, the beam intensity was monitored and measured in real time.

Table 1: Beam characteristics

\begin{tabular}{|c|c|c|c|}
\hline Beam & $\begin{array}{c}\text { Energy } \\
\text { (MeV/u) }\end{array}$ & $\begin{array}{c}\text { Irradiation } \\
\text { time (h) }\end{array}$ & $\begin{array}{c}\text { Average } \\
\text { intensity (pps) }\end{array}$ \\
\hline${ }^{36} \mathrm{~S}$ & 12 & 12 & $1 * 10^{12}$ \\
\hline${ }^{208} \mathrm{~Pb}$ & 6.25 & 15 & $1 * 10^{11}$ \\
\hline
\end{tabular}

\subsection{Analysis method for the activation foils}

\subsubsection{Measurement and unfolding method}

Two Ortec HPGe detectors were used to measure the $\gamma$-ray spectra from the activation foils. The measurements were performed successively and the ordering was chosen according to the half-life of each nuclide. Gamma-ray spectra were recorded using the Interwinner software and were analyzed manually with the Fityk program [6]. After the activity for each isotope of interest in the foils was determined, the corresponding reaction rate was calculated.

In this work, we developed an unfolding program to reconstruct the incident neutron spectrum. This program adopts the SAND-II algorithm that is coupled to an applied Monte-Carlo technique [7,8,9]. The input of the unfolding program requires reaction rates, cross sections for each isotope, as well as their relative uncertainties. The cross sections used were those from the evaluated neutron cross-section libraries EAF-2010 [10].

\subsubsection{Uncertainties}

There were three main sources of uncertainties:

1. The uncertainty on the measured activities is about $5 \%$ - $10 \%$ due mainly to the efficiency calibration of the HPGe detector.

2. The uncertainty of the beam intensity and total irradiation time was evaluated to be about $5 \%$ for these two experiments.

3. Uncertainties associated with the cross sections range from a few to up to $10 \%$ for high energy threshold reactions [10].

\section{Monte-Carlo simulation codes}

Two nuclear Monte-Carlo transport codes, PHITS and FLUKA, were used to simulate the neutron spectrum. Comparison to our experimental data then provides a means to benchmark the simulated results.

\subsection{FLUKA}

FLUKA (FLUktuierende KAskade) is a MonteCarlo transport code developed by INFN and CERN. In FLUKA, the Boltzmann Master Equation (BME) model is used to simulate the dynamic processes of reactions from ions lower than $100 \mathrm{MeV} / \mathrm{u}[11,12]$. Fluka also uses the Weisskopf-Ewing model to compute the resulting compound nuclei and their evaporation products $[11,12]$.

\subsection{PHITS}

PHITS (Particle and Heavy Ion Transport code System) is a Monte-Carlo code developed by a Japanese collaboration between JAEA, RIST, KEK and other institutes. This code uses the JAERI Quantum Molecular Dynamics (JQMD) model to simulate the dynamic processes of reactions. JQMD can be used to simulate induced heavy-ion reactions from $10 \mathrm{MeV} / \mathrm{u}$ to 3.5 $\mathrm{GeV} / \mathrm{u}$. A Generalized Evaporation Model (GEM) is adopted to simulate the evaporation of the compound nuclei [13].

\section{Results}

In this section, we compare the unfolded spectra to the simulated ones obtained from PHITS and FLUKA for neutrons emitted at $0^{\circ}$ with respect to the beam axis.

The unfolded spectra of the ${ }^{36} \mathrm{~S}(12 \mathrm{MeV} / \mathrm{u})+\mathrm{Cu}$ reaction and comparison to FLUKA and PHITS estimates are shown in Fig.2. In this experiment, the highest threshold reaction observed was at $40 \mathrm{MeV}$. Above this energy, threshold reactions were not statistically observable at this beam intensity $\left(10^{12} \mathrm{pps}\right)$. The uncertainty of unfolded spectra is about $15 \%$ below this threshold (40 MeV). 
For low neutron energies $(<20 \mathrm{MeV})$, FLUKA agrees rather well with the experimental spectrum while PHITS underestimates the experimental data by a factor of 2 . The JQMD model does not simulate heavy-ion reactions below $10 \mathrm{MeV} / \mathrm{u}$. This could explain why PHITS underestimates the low-energy neutrons that are mainly produced from low-energy heavy ions that slow down in the target.

At higher neutron energies, there is good agreement between FLUKA and PHITS. However, these calculations both overestimate the experimental data by up to a factor of 3 at $40 \mathrm{MeV}$.

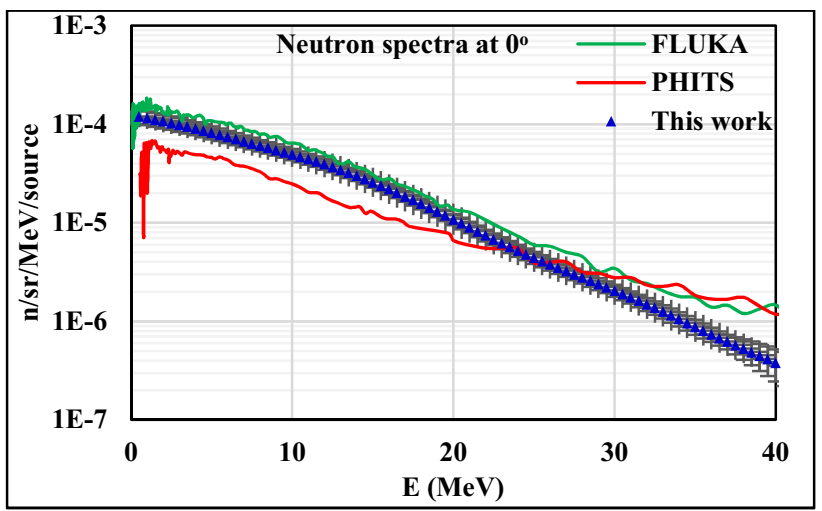

Figure 2: Unfolded spectrum of ${ }^{36} \mathrm{~S}(12 \mathrm{MeV} / \mathrm{u})+{ }^{\text {nat }} \mathrm{Cu}$ compared to FLUKA and PHITS simulations.

The unfolded spectrum of the second experiment $\left({ }^{208} \mathrm{~Pb}(6.25 \mathrm{MeV} / \mathrm{u})+{ }^{\text {nat }} \mathrm{Cu}\right)$ and its comparison to FLUKA and PHITS calculations is shown in Fig.3. In this experiment, the highest threshold reaction measured was about $15 \mathrm{MeV}$ due also to statistics. Uncertainties in this experiment were also about $15 \%$.

For this reaction FLUKA calculates a spectrum that is about 4 times larger than the experimental unfolded one. In this case, PHITS was not used to simulate this reaction because of its inherent limitations at low energies as described above.

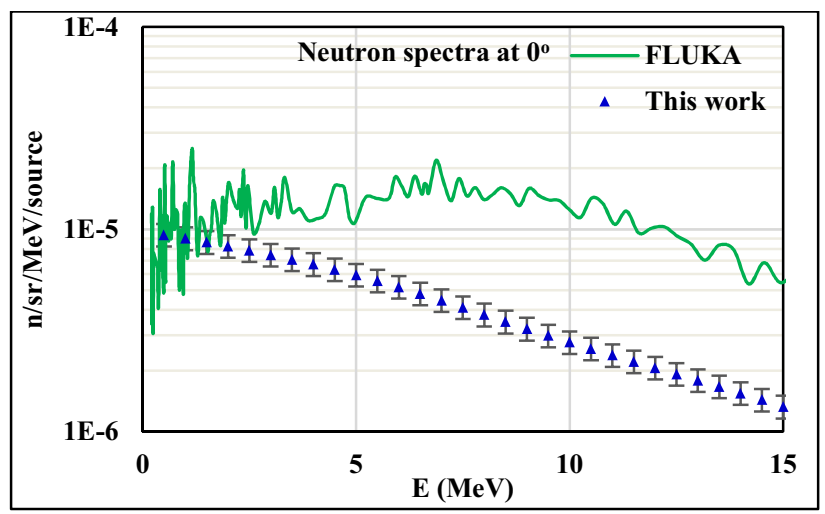

Figure 3: Unfolded spectrum of ${ }^{208} \mathrm{~Pb}(6.25 \mathrm{MeV} / \mathrm{u})+$ ${ }^{\text {nat }} \mathrm{Cu}$ compared to the FLUKA simulation.

These results show clear limitations of transport codes to estimate neutron yields from the interactions of low-energy and heavy ions, especially for high-energy neutrons.

\section{Conclusion}

In this work, we measured the double differential neutron spectrum from a thick ${ }^{\text {nat }} \mathrm{Cu}$ target bombarded by ${ }^{36} \mathrm{~S}(12 \mathrm{MeV} / \mathrm{u})$ and ${ }^{208} \mathrm{~Pb}(6.25 \mathrm{MeV} / \mathrm{u})$ beams using the foil-activation method. An unfolding program using the SAND-II algorithm coupled to a Monte-Carlo method has been developed to reconstruct the incident neutron spectra. Two Monte-Carlo transport codes (FLUKA and PHITS) were used to calculate the neutron spectrum and to benchmark them with experiment.

For the ${ }^{36} \mathrm{~S}(12 \mathrm{MeV} / \mathrm{u})+{ }^{\text {nat }} \mathrm{Cu}$ reaction, PHITS underestimates the results for low-energy neutrons $(<20$ $\mathrm{MeV}$ ) at $0^{\circ}$ while FLUKA seems to be in good agreement with the experiment. At higher energies, both codes agree with each other but systematically overestimate the experimental data by up to a factor of 3 .

For the ${ }^{208} \mathrm{~Pb}(6.25 \mathrm{MeV} / \mathrm{u})+{ }^{\text {nat }} \mathrm{Cu}$ reaction, the spectrum at $0^{\circ}$ calculated by FLUKA is higher than unfolded experimental one by up to a factor of 3 .

The results presented here are still preliminary and are being checked and improved by our team. After the final verification, we will apply the method described here to other angles. We are also studying the physics models at this low-energy limit for heavy ions in order to better understand the disagreement between calculation and experiment.

\section{References}

[1] C. Sunil et al, Thick target neutron yield from 145 $\mathrm{MeV}{ }^{19} \mathrm{~F}+{ }^{27} \mathrm{Al}$ system, Nuclear Instruments and Methods in Physics Research A 721 (2013) 21-25.

[2] V. Suman et al, Thick target double differential neutron energy distribution from ${ }^{12} \mathrm{C}+{ }^{27} \mathrm{Al}$ at $115 \mathrm{MeV}$, Nuclear Instruments and Methods in Physics Research A 800 (2015) 29-33.

[3] G. Lhersonneau et al, Neutron yield from carbon, light- and heavy-water thick targets irradiated by $40 \mathrm{MeV}$ deuterons, Nuclear Instruments and Methods in Physics Research A 603 (2009) 228-235.

[4] G. Lhersonneau et al, A facility for fast-neutron irradiations at Jyväskylä and its use for nuclide crosssection measurements in fission, Nuclear Instruments and Methods in Physics Research A 698 (2013) 224-233.

[5] G. F. Knoll, Radiation Detection and Measurement Third edition, John Wiley \& Sons, Inc, 2000.

[6] M. Wojdyr, Fityk: a general-purpose peak fitting program, J. Appl. Cryst. (2010). 43, 1126-1128.

[7] W. N. McElroy et al, SAND-II Neutron Flux Spectra Determinations by Multiple Foil Activation Iterative Method. RSIC Computer Code Collection CCC-112. Oak Ridge National Laboratory, Information Center, May 1969. 
[8] É. M. Zsolnay and E. J. Szondi, Neutron spectrum determination by multiple foil activation method, Nuclear Training Reactor of the Technical University Budapest, 1982.

[9] S. Maeda et al, Fundamental Study on Neutron Spectrum Unfolding using Maximum Entropy and Maximum Likelihood Method, Progress in Nuclear science and technology, Vol. 1, p.233-236 (2011).

[10] J.-Ch. Sublet et al, The European Activation File:

EAF-2010 neutron-induced cross section library, EASY Documentation Series CCFE-R (10) 05

[11] A. Ferrari et al, FLUKA: a multi-particle transport code, CERN-2005-10 (2005), INFN/TC_05/11, SLAC-R773.

[12] T.T. Böhlen et al, The FLUKA Code: Developments and Challenges for High Energy and Medical Applications, Nuclear Data Sheets 120, 211-214 (2014).

[13] T. Sato et al, Particle and Heavy Ion Transport Code System PHITS, Version 2.52, J. Nucl. Sci. Technol. 50:9, 913-923 (2013). 